Editorial

\title{
Trabajo y salud respiratoria
}

\section{Work and respiratory health}

\section{Pere Casan Clarà}

Hospital Universitario Central de Asturias. Facultad de Medicina. Oviedo. España.

Recibido: 25-10-13

Aceptado: 31-10-13

\section{Correspondencia}

Pere Casan Clarà

Instituto Nacional de Silicosis

Área del Pulmón

Hospital Universitario Central de Asturias

Facultad de Medicina

Universidad de Oviedo

Oviedo. España.

Correo electrónico: pcasan@ins.es

\section{ResUmen}

Las enfermedades respiratorias son una parte importante de la patología laboral y medioambiental. Si el aire que respiramos está contaminado el pulmón es el órgano diana de los efectos del tabaco, sílice, asbesto, antígenos diversos, $\mathrm{NO}_{2}, \mathrm{SO}_{2}$, nanopartículas, etc. Los organismos responsables de la salud comunitaria deben velar para que el diagnóstico, prevención y tratamiento de estos procesos sea el más adecuado. El Instituto Nacional de Silicosis y el Instituto de Salud Carlos III son, con su actuación, un buen ejemplo de esta actitud.

Med Segur Trab (Internet) 2013; 59 (233) 372-374

Palabras clave: Neumología ocupacional, silicosis, asbestosis, salud respiratoria.

\begin{abstract}
The respiratory diseases are an important part of the occupational and environmental pathology. If the air that we breathe is contaminated the lungs are the target organ of the tobacco, silica, asbestos, antigens, $\mathrm{NO}_{2}, \mathrm{SO}_{2}$, nano-particles effects. The agencies responsible for community health must ensure that the diagnosis, prevention and treatment of these processes are the most appropriate. The Instituto Nacional de Silicosis and the Instituto de Salud Carlos III are with this action a good example of this attitude.
\end{abstract}

Med Segur Trab (Internet) 2013; 59 (233) 372-374

Key words: Occupational Pneumology, silicosis, asbestosis, respiratory bealth. 
Comer, beber, dormir, respirar..., forman parte de la base de la pirámide que el psicólogo norteamericano Abraham Maslow diseñó en 1943, en su famoso tratado sobre la jerarquía de las necesidades humanas ${ }^{1}$. Con la respiración diaria entran y salen de los pulmones aproximadamente unos 5.000 litros de aire. Con el aire, el oxígeno, y con éste la necesaria y temible oxidación de los tejidos. Afortunadamente disponemos de mecanismos homeostáticos para reducir este efecto de los radicales libres y si no fuera por elementos ajenos que entran junto al aire, nuestros pulmones estarían preparados para sobrevivir casi una centuria.

Trabajar se sitúa en el segundo piso de la pirámide mencionada y, paradojas de la época, todos estaríamos de acuerdo en que se situara también en la base. Sin ánimo de entrar en otras polémicas que no corresponden a este editorial, aproximadamente 1/6 de nuestra vida lo pasamos en el trabajo, de lo que se deduce el interés en que el "aire laboral" sea lo más puro posible. Afortunadamente las leyes nos protegen de uno de los mayores peligros aceptados que contaminan el aire que respiramos. Me refiero al tabaco, y todo esfuerzo es poco para mantener en positivo unas conquistas que tantas energías han costado a la sociedad.

Pero cuando hablamos de trabajo y salud respiratoria, generalmente nos referimos a contaminantes muy conocidos y que en el pasado reciente han sido motivo de gran interés público. La sílice, cuyo contenido en la corteza terrestre es de más del 25\%, más común en las minas de interior (carbón) pero muy presente en canteras de superficie (pizarras, granito, etc) ; el amianto, muy utilizado como elemento de materiales de construcción y aislamiento térmico (prohibido desde el año 2002) ; antígenos presentes en el ámbito laboral de ciertas profesiones, etc. Aunque algunos de estos elementos han sido cada día mejor y más controlados y ya no ocupan la cabecera de las publicaciones médicas, su presencia es activa en nuestra vida y no podemos bajar la guardia sobre su potencial nocividad ${ }^{2}$.

El estudio, diagnóstico, prevención y tratamiento de las enfermedades respiratorias de origen laboral y medioambiental ha sido siempre un área de interés de la Neumología. Interés compartido, por otra parte, por diferentes instituciones y servicios. El Sistema Nacional de Salud, a través de sus organismos y representaciones, los diferentes Servicios de Prevención de Riesgos Laborales, Mutualidades Sanitarias, Unidades de Valoración, etc. han hecho y hacen de la salud respiratoria de todos los trabajadores un objetivo común. Y debemos decir con orgullo que este objetivo se ha cumplido y se cumple de forma más que correcta. Cómo si no, se habrían reducido hasta las cifras actuales la tuberculosis, la silicosis, el asma, incluso el propio tabaquismo, sin la actuación firme y decidida de todos estos organismos?. El diagnóstico precoz, las medidas preventivas y los recursos técnicos aplicados a la minería del carbón hicieron que la silicosis casi desapareciera de este ámbito laboral antiguamente tan sensible. Los cuidados higiénicosanitarios y el uso adecuado de fármacos obraron el milagro de controlar la tuberculosis.... Como decía, podemos disponer actualmente de un buen "haber" ganado a pulso entre todos nosotros.

Pero, un nuevo reto se abre ante todos los responsables de la salud respiratoria en el medio laboral. No podemos bajar la guardia sobre los agentes de siempre (tabaco), pero debemos mantener las antenas bien altas sobre nuevas causas y nuevas enfermedades. La silicosis se abre en otros frentes: las canteras a cielo abierto, donde la piedra ornamental o de triturado, tan útiles en la construcción de edificios o carreteras, se manipula sin las debidas precauciones; la colocación, sin las condiciones adecuadas, de conglomerados de cuarzo que contienen polvo miscroscópico de sílice cristalina ${ }^{3}$; el desguace de edificios donde el asbesto formaba parte de la estructura ${ }^{4}$; nuevas profesiones o las de siempre, donde se inhalan antígenos originados en pinturas, tintes, maderas, pigmentos, etc ${ }^{5}$; inhalación de partículas en suspensión fruto de la combustión de materia orgánica, con radicales libres de ozono, $\mathrm{SO}_{2} \mathrm{O} \mathrm{NO}_{2}{ }^{6}$; partículas radioactivas originadas en el subsuelo (radón) ${ }^{7}$; las denominadas nanopartículas procedentes de industrias químicas y de transformación, etc. 
La prevención es siempre la mejor medida para resolver un problema sanitario. Anticiparnos a los síntomas, al ingreso hospitalario, a la pensión compensatoria, al sufrimiento,...es rentable desde todos los puntos de vista, tanto económico como social. Pero todos sabemos lo difícil que resulta modificar nuestros hábitos de conducta, especialmente los que crean adicción, como nos resistimos a poner la mascarilla, a vacunarse de la gripe, a evitar los agentes tóxicos que nos rodean. Por este motivo es tan importante que el ejemplo personal y colectivo, en nuestros ámbitos de responsabilidad, sea el mejor activo para favorecer la prevención.

El diagnóstico y tratamiento de las enfermedades respiratorias de origen laboral y medioambiental corresponde a los neumólogos y a los médicos del trabajo, que contarán con el apoyo de los primeros en cuantas cuestiones sean necesarias (pruebas funcionales, exploraciones complejas, evaluaciones específicas). La evaluación médico legal puede contar también con Unidades o Servicios con elevada tradición y especialización. En nuestro caso, el Instituto Nacional de Silicosis (INS), dispone de la preparación y la experiencia necesarias para confirmar o descartar patologías respiratorias que, por su prevalencia, han ocupado y ocupan una parte importante de nuestra actividad. Ponemos también a disposición de aquellos organismos que lo consideren nuestra experiencia docente e investigadora para colaborar en mantener activa la llama de la formación en todos estos aspectos comentados. Por poner un ejemplo de potenciación de "institutos", el INS colabora ya con el Instituto de Salud Carlos III en programas de formación específicos en la Neumología Ocupacional.

Soplan vientos de cambio en la vieja Europa. Vientos que arrastran partículas que pueden originar el "efecto mariposa". Algunos países orientales con elevados niveles de crecimiento emiten partículas a la atmósfera que traerán tempestades aún desconocidas. Tal como hiciera Ulises al amarrarse al palo mayor para no caer bajo el embrujo de las sirenas, deberemos estar muy atentos para no sucumbir a este torrente de calamidades que algunos agoreros nos anuncian. Sólo nuestra preparación y nuestro trabajo nos permitirán seguir en la brecha para atender las necesidades sanitarias que este convulso siglo XXI nos depare.

\section{REFERENCIAS BIBLIOGRÁFICAS}

1. Maslow A.H. A theory of human motivation. Psychological Review 1943;50:370-396.

2. Martínez C ; Prieto A ; García L ; Quero A ; González S ; Casan P. Silicosis, una enfermedad con presente activo. Arch Bronconeumol 2010;46:97-100.

3. García C ; Sánchez J ; Romero J. Silicosis en trabajadores de conglomerados de cuarzo. Arch Bronconeumol $2011 ; 47: 53$

4. Ferrer J ; Martínez C. El diagnóstico de las enfermedades respiratorias causadas por el asbesto. Arch Bronconeumol 2008;44:177-179.

5. Morell F ; Villar A ; Montero MA ; Muñoz X ; Colby TV ; Pipvath S ; Cruz MJ ; Raghu G. Chronic hypersensitivity pneumonitis in patients diagnosed with idiopathic pulmonary fibrosis: a prospective cas-cohort study. The Lancet Respiratory Medicine 2013 doi:10.1016/S2213-2600(13)70191-7.

6. McCreanor J ; Cullinan P ; Nieuwenhuijsen MJ. et al. Respiratory effects of exposure to dieses traffic in persons with asthma. N Eng J Med 2007;357:2348-2358.

7. Ruano-Ravina A ; Barros-Dios JM. Radón y cancer de pulmón. Implicaciones para profesionales sanitarios, ciudadanos y administraciones públicas. Medicina Clínica. Barcelona 2007;128:545-549.

8. Raaschou-Nielsen O ; Andersen ZJ ; Beelen B. et al. Air pollution and lung cancer incidence in 17 European cohorts: prospective analyses from the European Study of cohorts for air pollution effects (ESCAPE). The Lancet Oncology 2013;14:813-822. 